\title{
Treatment Options for Central Retinal Artery Occlusion
}

Sudha Cugati, MS, $P h D^{1}$

Daniel D. Varma, $B M B S^{2}$

Celia S. Chen, MBBS, PhD, FRANZCO $0^{3, *}$

Andrew W. Lee, MBBS, MPH, FRACP

\author{
Address \\ ${ }^{1}$ Department of Ophthalmology, University of Adelaide, Adelaide, SA 5000, \\ Australia \\ Email: sudhacugati@gmail.com \\ ${ }^{2}$ Flinders Comprehensive Stroke Centre, Flinders Medical Centre, Bedford Park, \\ SA 5042, Australia \\ Email:varm0006@flinders.edu.au \\ *,30partment of Ophthalmology, Flinders Medical Centre and Flinders University, \\ Flinders Drive, Bedford Park, SA 5042, Australia \\ Email: Celia.Chen@health.sa.gov.au \\ ${ }^{4}$ Flinders Comprehensive Stroke Centre, Flinders Medical Centre, Bedford Park, \\ SA 5042, Australia \\ Email: andrew.lee@health.sa.gov.au \\ Published online: 16 October 2012 \\ (C) The Author(s) 2012. This article is published with open access at Springerlink.com
}

Keywords Central retinal artery occlusion - Treatment - Thrombolysis - Thrombolytic therapy - Vasodilators • Intraocular pressure $\cdot$ Neovascularization

\section{Opinion statement}

Central retinal artery occlusion (CRAO) is an ocular emergency and is the ocular analogue of cerebral stroke. It results in profound, usually monocular vision loss, and is associated with significant functional morbidity. The risk factors for CRAO are the same atherosclerotic risk factors as for stroke and heart disease. As such, individuals with CRAO may be at risk of ischemic end organ damage such as a cerebral stroke. Therefore, the management of CRAO is not only to restore vision, but at the same time to manage risk factors that may lead to other vascular conditions. There are a number of therapies that has been used in the treatment of CRAO in the past. These include carbogen inhalation, acetazolamide infusion, ocular massage and paracentesis, as well as various vasodilators such as intravenous glyceryl trinitrate. None of these "standard agents" have been shown to alter the natural history of disease definitively. There has been recent interest shown in the use of thrombolytic therapy, delivered either intravenously or intra-arterially by direct catheterisation of the ophthalmic artery. Whilst a number of observational series have shown that the recovery of vision can be quite dramatic, two recent randomised controlled trials have not demonstrated efficacy. On the contrary, intra-arterial delivery of thrombolytic may result in an increased risk of intracranial and systemic haemorrhage, while the intravenous use of tissue plasminogen activator (tPA) was not shown to be efficacious within $24 \mathrm{~h}$ of symptom onset. Nevertheless, both of these studies have shown one thing in common, and that is for treatment 
to be effective in CRAO, it must be deployed within a short time window, probably within $6 \mathrm{~h}$ of symptom onset. Therefore, while CRAO is a disease that does not have a treatment, nevertheless it needs to follow the same principles of treatment as any other vascular end organ ischaemic disease. That is, to attempt to reperfuse ischemic tissue as quickly as possible and to institute secondary prevention early.

\section{Introduction}

Central retinal artery occlusion (CRAO) is the occlusion of the central retinal artery (CRA) with resultant infarction of the retina and vision loss. It was first described as an embolic occlusion of the CRA in a patient with endocarditis by von Graefe in 1859 [1]. The incidence of CRAO is estimated around 1.9/100,000 in the United States [2・].

Patients with CRAO typically present with an acute, painless loss of vision, and $80 \%$ of affected patients have a final visual acuity of counting fingers or worse $[3,4]$. Visual loss in CRAO occurs as a result of loss of blood supply to the inner retinal layers. Approximately 15$30 \%$ of the general population have a cilioretinal artery, which is a branch of the short posterior ciliary artery [5]. It supplies a part or the whole of the fovea, and in those eyes where there is a CRAO, the cilioretinal artery is spared and the visual acuity may be preserved at 20/50 or better, with loss of peripheral vision only.

The most common cause of CRAO is thromboembolus, which occurs at the narrowest part of the central retinal artery, where it pierces the dural sheath of the optic nerve $[6 \bullet \bullet, 7]$. It could also occur as a result of an occlusive thrombus at the level immediately posterior to the lamina cribrosa [8]. Once the central retinal artery is occluded, the ability of the retina to recover depends on whether the offending embolus or thrombus is dislodged, and also on the retinal tolerance time $[9,10]$.

CRAO is divided into four distinct clinical entities:

(1) Non-arteritic permanent CRAO (Fig. 1)

This group accounts for over two thirds of all CRAO cases, and is caused by platelet fibrin thrombi and emboli as a result of atherosclerotic disease [11-13].

(2) Non-arteritic transient CRAO

Non-arteritic transient CRAO (transient monocular blindness) accounts for $15 \%$ of CRAOs and has the best visual prognosis. People suffering from this have a $1 \%$ risk per year of having a permanent non-arteritic CRAO [14]. Transient vasospasm due to serotonin release from platelets on atherosclerotic plaques has also been suggested as a mechanism of transient CRAO in animal models [9].

(3) Non-arteritic CRAO with cilioretinal sparing (Fig. 2)

Preservation of the cilioretinal artery results in preserved perfusion of the macula region [4].

(4) Arteritic CRAO (Fig. 3)

Arteritic CRAO (including the vasculitides) accounts for $<5 \%$ of cases. Giant cell arteritis is the most common entity in this category, and can cause bilateral visual loss. If an arteritic cause is suspected, it is essential to assess the inflammatory markers and treat urgently with systemic corticosteroids.

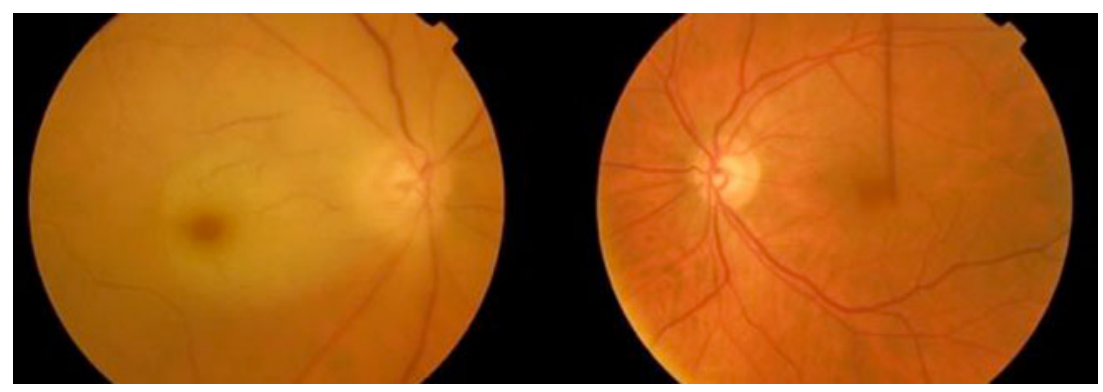

Figure 1. Colour fundus photograph showing non-arteritic permanent central retinal artery occlusion in the right eye and a normal fundus in the left eye. 


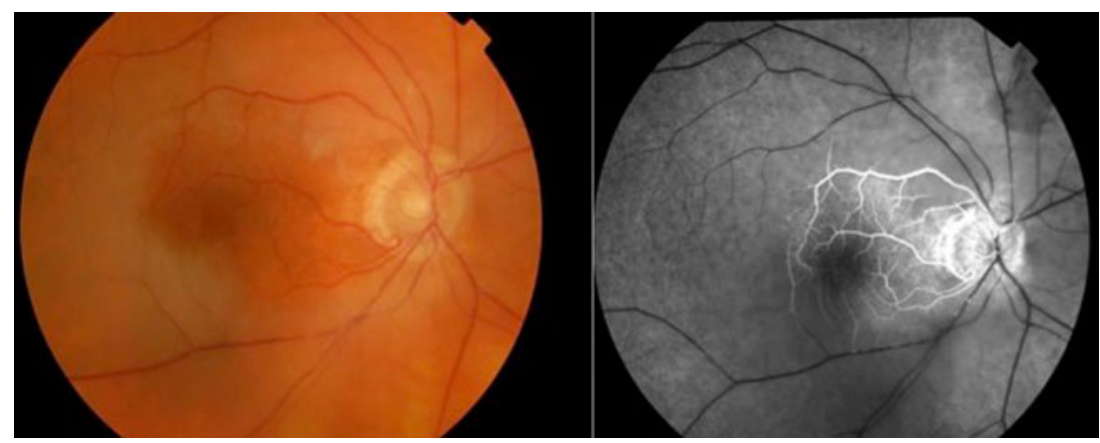

Figure 2. Colour fundus photograph and fundus fluorescein angiogram of the right eye showing non-arteritic CRAO with cilioretinal sparing.

The majority of CRAOs are of the non-arteritic permanent type and are analogous to a terminal arterial branch occlusion in cerebral stroke. In a suspected non-arteritic CRAO due to thrombo-embolic cause, evaluation of atherosclerotic risk factors, including a family history of cerebrovascular and cardiovascular disease, diabetes mellitus, hyperlipidemia, a past history of vascular disease, smoking, palpitations, valvular heart disease or transient ischaemic events such as transient monocular blindness, transient ischemic attack (TIA) or angina symptoms, should be sought
[15-22]. In those CRAOs where there are no atherosclerotic risk factors, especially in a young patient, other less common factors should be explored. These include the presence of vasculitis, sickle cell disease, myeloproliferative disorders, hypercoagulable states, and the use of the oral contraceptive pill or illicit drugs [23].

The following discussion on therapy concentrates mainly on the management of non-arteritic CRAO of presumed thromboembolic origin that forms the majority of CRAO cases.

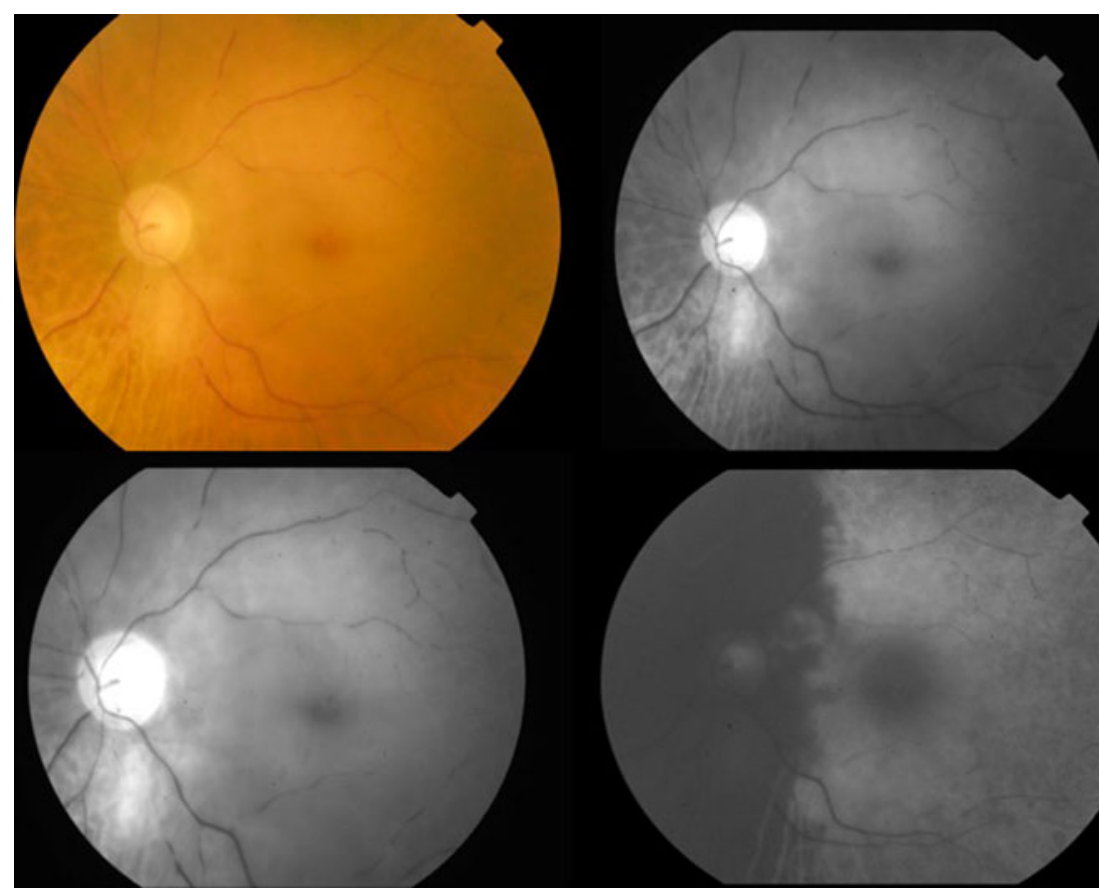

Figure 3. Colour fundus photograph of the left eye showing arteritic central retinal artery occlusion; serial fundus fluorescein angiogram showing delayed arterial filling and choroidal ischaemia. 


\section{Treatment}

\section{Diet \& lifestyle}

\section{Pharmacological treatment}

The risk factors for CRAO are those of atherosclerosis [24, 25]. Diet and lifestyle play a role indirectly for secondary prevention of further end organ ischemia. Hypertension and diabetes are the most frequent risk factors for CRAO [24]. Other associated risk factors include smoking, hypercholesterolemia and a family history of microvascular disease.

As a result, a diet with a low glycemic index would reduce the risk of vascular disease [26]; such a diet is rich in fruits, vegetables, grains, lowfat or nonfat dairy products, fish, legumes, poultry, and lean meats, polyunsaturated fatty acids. Also, regular exercise is essential to reduce the cardiovascular risk factors [27]. A healthy diet hence plays a role for secondary prevention in CRAO.

Hyperhomocystinemia is also recognised as a risk factor for CRAO [28], and results in dysfunction of vessel endothelium, with a proliferation of vascular smooth muscle and prothrombic hemostatic changes. Homocysteine levels are known to increase in a diet deficient in folic acid and vitamin B6 and B12, and hence diet with adequate folic acid and vitamin B6 and B12 in patients with hyperhomocystinemia would reduce the risk of CRAO.

The retinal tolerance time to acute ischaemia has been evaluated in experimental studies [29]. These studies suggest that the retina in elderly, atherosclerotic and hypertensive rhesus monkeys suffer no detectable damage to CRAO for $97 \mathrm{~min}$. Thereafter, partial recovery is possible if the ischemia is reversed up to $240 \mathrm{~min}$. The time when irreversible retinal damage occurs with no recovery of vision is not known, but postulated to be around 6 to $6.5 \mathrm{~h}[30 \bullet, 31]$. Hence, for any treatment to be effective, it is essential to implement the treatment within the correct time window. Spontaneous recanalization of the occluded central retinal artery may occur within $48 \mathrm{~h}$ to $72 \mathrm{~h}$, but this may be partial [3]. Current reports on the visual improvement rate vary from $1 \%$ to $10 \%$ of cases of non-arteritic CRAO [13, 32]. Recent studies have suggested that the minimally invasive therapy in CRAO do not result in a significant visual recovery $[33,34]$.

Table 1 summarises the various available pharmacological options in the treatment of CRAO.

\section{Vasodilators, which help in increasing the blood oxygen content}

Pentoxyphylline [35, 36]

Pentoxyphylline is a tri-substituted xanthine derivative that works by increasing erythrocyte flexibility, reducing blood viscosity, and increasing microcirculatory flow and tissue perfusion. It has been used in the treatment of peripheral vascular disease. In a randomised control trial, a small number of patients (ten patients) with CRAO were randomised to either pentoxiphylline or placebo for 4 weeks. The endpoint measurements include objective 


\section{Table 1. Options available in treatment of central retinal artery occlusion}

\section{Group of drugs}

1. Vasodilators

Pentoxyphylline

Inhalation of carbogen

Hyperbaric oxygen

Sublingual isosorbide dinitrite

2. Ocular massage

Anterior chamber paracentesis

Intravenous acetazolamide

Intravenous mannitol

Topical antiglaucoma

medications

3. Intravenous methylprednisolone

4. Nd YAG Laser embolectomy

5. Intra arterial or intravenous thrombolysis

\section{Mechanism of action}

Increase the blood oxygen content

Reduce intraocular pressure and hence increase the retinal artery perfusion or help dislodge the embolus

Reduction of retinal oedema Lyse or dislodge the clot Help in thrombolysis of the embolus
Standard dosage

Contra indications

Main drug interactions

Main side effects

Cost / cost effectiveness
CRA blood flow measured by duplex scanning. The authors noted an increase in the peak systolic and end diastolic flow velocities by $550 \%$ and $400 \%$, respectively, in five patients treated with pentoxifylline, versus $288 \%$ and $200 \%$, respectively, in a placebo group of five patients. However, the visual recovery was not discussed in the study and the numbers were small [36].

$600 \mathrm{mg}$ tds

Allergy to theophylline or caffeine

Warfarin: Pentoxiphylline increases the anticoagulant effect of warfarin Theophylline \& Aminophylline: When used in combination, pentoxiphylline potentiates the effect of theophylline and aminophylline

Digestive (dry mouth or dehydration, constipation, anorexia, cholecystitis) Neurogenic (aseptic meningitis, seizures, confusion, depression anxiety),

Cardiovascular (hypotension, edema, dyspnea)

Respiratory (nasal congestion, nosebleed, breathing difficulty)

Dermatological (rash, angioedema, urticaria, pruritus, brittle fingernails)

Ear and eye related (earache, scotoma, conjunctivitis, blurred vision)

Pentoxyphylline is inexpensive, but the effectiveness is not established.

Carbogen is a mixture of 4-7 \% carbon dioxide and 93-96\% oxygen, used in the treatment of CRAO based on the assumption that carbon dioxide will prevent oxygen-induced vasoconstriction and hence maintain or even improve the blood flow while maintaining the oxygenation of the retina $[37,38]$. Carbogen inhalation is conducted for 10 min every hour during waking hours, and 10 min every $4 \mathrm{~h}$ at night and continued for 48-72 h. However, retinal dynamics obtained with carbogen have 
been contradictory [39], and the results of visual recovery with carbogen in acute CRAO was not significant [40].

The proposed role for hyperbaric oxygen in CRAO is an increase the partial pressure of oxygen delivery to ischemic tissue until spontaneous or assisted reperfusion occurs. The exact pathogenesis is debated $[41,42,43 \bullet, 44-46]$ and the efficacy is not proven. The protocol for hyperbaric oxygen varies in different studies, with an average of 2-2.5 atm for approximately $90 \mathrm{~min}$ within $8 \mathrm{~h}$ of onset of CRAO [47].

Sublingual isosorbide dinitrite

\author{
Standard dosage \\ Contraindications \\ Main drug interactions
}

Main side effects

Cost / cost effectiveness
Nitroglycerin causes relaxation of vascular smooth muscle by stimulating intracellular cyclic guanosine monophosphate (GMP). Nitroglycerine has been used in central retinal artery occlusion along with other modes of treatment, including ocular massage and other means to reduce the intraocular pressure [48].

$10 \mathrm{mg}$

Hypersensitivity, severe anaemia, recent use of phosphodiesterase inhibitors Drugs which decrease the effect of isosorbide dinitrite include:

- Dopamine receptor agonist (Bromocriptine, cabergoline)

- Ergopeptides (dihydroergotamine, ergotamine, methylergonovine, methysergide)

- Drugs which have an additive vasodilator effect

- Phosphodiesterase inhibitors

Common: Headache, hypotension, tachycardia, dizziness, lightheadedness, blurred vision, flushing, nausea and vomiting, nervousness, xerostomia Serious: Methemoglobinemia (rare), syncope, prolonged bleeding time, exfoliative dermatitis, unstable angina, rebound hypertension, thrombocytopenia

Isosorbide dinitrate is inexpensive, but the effectiveness is not established.

\section{Reduction of intraocular pressure, and hence increase the retinal artery perfusion or help dislodge the embolus}

As mean ocular perfusion pressure is the difference between mean arterial pressure and intraocular pressure, attempts have been made to reduce the intraocular pressure and thus increase ocular perfusion [33, 48].

Ocular massage includes the compression of the globe with a threemirror contact lens for $10 \mathrm{~s}$, to obtain retinal artery pulsation or flow cessation if the pulsation is not seen followed by a 5 s release $[16,48$, 49]. Alternatively, digital massage can be applied over the globe over the closed eyelids for 15-20 min. The combination of the massage and acetazolamide can decrease the intraocular pressure to as low as 
$5 \mathrm{mmHg}$ within a short period of time [49]. Ocular massage causes retinal arterial dilatation and large fluctuations in Intraocular pressure (IOP). It has been postulated that this activity may mechanically facilitate the disintegration of a thrombus, or dislodge an impacted embolus into a more peripheral part of the retinal circulation [18].

Standard dosage

Contraindications

Main drug interactions

Main side effects

Cost / cost effectiveness
Acetazolamide is a carbonic anhydrase inhibitor that reduces the aqueous production and hence reduces the intraocular pressure. This in turn increases the retinal perfusion [48].

intravenous injection 500 stat or $250 \mathrm{mg}$ qid for $24 \mathrm{~h}$

Hypokalemia \& hyponatremia, hyperchloremic acidosis, sulfa allergy, liver or renal disease including liver cirrhosis

Methenamine: When methenamine is used with acetazolamide, there are insoluble precipitates in the urine, and this decreases the effect of both drugs Cisapride: Acetazolamide increases the toxicity of cisapride by passive tubular resorption by increasing the $\mathrm{pH}$

CNS: Confusion, Convulsions, Drowsiness, Flaccid paralysis, Malaise GI: Anorexia, Diarrhea, Metallic taste, Nausea, Vomiting, Hepatic disease, Melena

Blood: Aplastic anemia, Agranulocytosis, Leukopenia Paresthesia, Thrombocytopenia, Thrombocytopenic purpura

Renal: Hematuria, Polyuria,

Electrolyte imbalance, Glycosuria, acidosis

Photosensitivity, Urticaria

Hearing dysfunction or tinnitus

Sulfonamide type reaction

Special points The medication is used as an immediate intravenous or short-term intensive oral therapy but not designed as a long-term therapy. Hence, the long-term monitoring in acetazolamide use do not apply in this setting.

Acetazolamide is inexpensive.
Standard dosage

Contraindications

Main drug interactions

Main side effects

Cost / cost effectiveness

\section{$1.5-2 \mathrm{~g} / \mathrm{Kg}$ over 30-60 min}

Hypersensitivity, severe dehydration, anuria, progressive renal disease, severe pulmonary edema or heart failure, metabolic edema, active intracranial bleeding

Tobramycin: Mannitol increases the level of tobramycin by unknown mechanism

Lurasidone, Nitroglycerine: these can increase the effect of mannitol

GI: Nausea, Vomiting

CVS: Angina-like chest pains, hypotension, congestive cardiac failure, Phlebitis CNS: Convulsions, Dizziness,

Electrolyte imbalances, acidosis

Blurred vision

Renal: Urinary retention

Mannitol is inexpensive. 
Topical antiglaucoma drops

Glaucoma medications aim to lower the intraocular pressure and increase the gradient across the optic nerve head (perfusion pressure= mean arterial pressure - intraocular pressure). The drops act by decreasing aqueous humor production (with beta-adrenergic blockers or carbonic anhydrase inhibitors), or increasing outflow (with prostaglandin analogue or an alpha-agonist). However, the onset of action is slow with topical drops compared to intravenous acetazolamide and mannitol.

\section{Reduction of retinal oedema}

\section{Intravenous methylprednisolone}

A single dose of intravenous methylprednisolone of $1 \mathrm{~g}$ has been reported in a case series of patients whose vision failed to improve following the conventional treatment of measures to reduce the intraocular pressure $[50,51]$. The possible mechanism of action is reported to be a reduction in the retinal oedema, and hence, improvement of vision. However, the patients were younger, and it is possible that these patients had vasospasm or CRAO secondary to vasculitis, rather than atherosclerotic-induced CRAO.

Special consideration Intravenous methylprednisolone is given in suspected arteritic CRAO due to giant cell arteritis, but is not a recommended treatment for non-arteritic permanent CRAO.

\section{Thrombolytic therapy}

Tissue plasminogen activator (tPA)

Tissue plasminogen activator (tPA) is a naturally occurring fibrinolytic agent found in vascular endothelial cells and results in clot lysis. At the site of the thrombus, the binding of tPA and plasminogen to the fibrin surface induces a conformational change that facilitates the conversion of plasminogen to plasmin and dissolves the clot.

Alteplase, a recombinant tPA, is a fibrin- specific agent commonly used in ischaemic stroke, myocardial infarction and massive pulmonary embolism. CRAO is a stroke of the eye analogous to ischemic cerebral stroke, and the CRA and retina are homologous to the circle of Willis and the brain respectively. Fibrinolysis is an accepted standard therapy in the treatment of ischaemic stroke, and hence there is biological plausibility for its use in CRAO.

Two large reviews [52, 53] and several observational studies [31, 54, 55] have suggested that thrombolysis in the treatment of CRAO may improve the visual acuity with few serious complications. However, data from a large

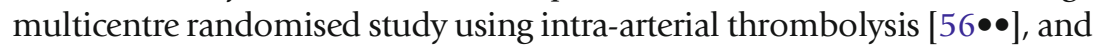
another randomised study using intravenous thrombolysis [30 $[$ ] have failed to show an improvement in the visual acuity. 
Specific drugs Various thrombolytic agents have been indicated in different studies. Most often, tPA or urokinase are used.

Standard dosage Intravenous tPA dose varied from a loading dose of $50 \mathrm{mg}$ infusion over $60 \mathrm{~min}$ (a dose smaller than recommended in ischaemic stroke) to $0.9 \mathrm{mg} / \mathrm{Kg}$ (maximum of $90 \mathrm{mg}$ ) that is the standard dose used in ischemic stroke [59]. The use of adjuvant therapy, such as heparin with intravenous heparin, or anti-platelet therapy is not standardized, and was greatly varied between the various studies to allow comparison and comment.

Contraindications [61]

- Evidence of intracerebral hemorrhage on pre-treatment evaluation

- Suspicion of subarachnoid hemorrhage on pretreatment evaluation

- Myocardial infarction, Intracranial or intraspinal surgery, serious head trauma or stroke in the last 3 months

- Gastro-intestinal or genito-urinary haemorrhage in the previous 3 weeks

- Early ischaemic changes occupying greater than one-third of the middle cerebral artery (MCA) territory

- History of intracerebral hemorrhage (ICH)

- Uncontrolled hypertension at time of treatment (>185 mmHg systolic or $>110 \mathrm{mmHg}$ diastolic)

- Blood glucose $>22.22 \mathrm{mmol}$ [62]

- NIH Stroke scale score $>25$ [62]

- Seizure at onset of stroke

- Active internal bleeding

- Intracranial neoplasm, arteriovenous malformation, or aneurysm

- Known bleeding diathesis, including but not limited to :

- Current use of oral anticoagulants such as Warfarin or an International normalised ratio (INR) of $>1.7$, prothrombin time (PT) of $>15 \mathrm{~s}$

- Administration of heparin within $48 \mathrm{~h}$ preceding the onset of the stroke and a elevated activated partial thromboplastin time (aPTT) at presentation

- Platelet count $<100,000 \mathrm{~mm}^{2}$ 
Main drug interactions [61]

Main side effects [61]

Special considerations

Cost / cost effectiveness
Increased risk of bleeding with the concomitant or immediate prior use of anti-coagulant, anti-platelet or vitamin $\mathrm{K}$ antagonists.

No studies on interactions of tPA and other cerebroactive drugs.

Intracerebral and systemic haemorrhage

The following have been reported in clinical trials and post marketing experience involving tPA: anaphylactoid reaction, laryngeal edema, orolingual angioedema (often due to concomitant use of ace-inhibitors), rash and urticaria.

Specific to ischaemic stroke: cerebral edema, cerebral herniation, seizure, new ischaemic stroke.

The role of adjuvant therapy with thrombolysis is not known. There is no consensus with regard to the standard dose, route of administration or simultaneous use of heparin in various studies. The EAGLE study used intraarterial tPA within $24 \mathrm{~h}$ of visual loss followed by heparin for 5 days [60].

The agent tPA cost approximately USD $\$ 1,487.06$ per $50 \mathrm{mg}$ vial and $\$ 2,974.13$ per $100 \mathrm{mg}$ vial (including diluent) [63]. It requires specialized neuro-intervention set-up and neuroradiologist for intra-arterial administration, or specialized stroke unit set-up for intra-arterial administration. Cost effectiveness has not been established in CRAO. However, a recent systematic review in The American Journal of Managed Care on cerebral ischaemic stroke has built upon primary cost effectiveness data from Fagan and colleagues in $1998[64,65]$. The study used data from the NINDS rt-PA Study [66] and medical literature to estimate health and economic outcomes in ischaemic stroke patients treated with rt-PA. A Markov model was then developed to estimate costs per 1,000 patients eligible for rt-PA vs. costs per 1,000 people untreated [64]. The estimated impact of rt-PA use on long term health outcomes was a savings of 564 quality-adjusted life years (QALYs) over 30 years in the 1,000 person model [64].

Cost effective calculations showed rt-PA treatment resulted in incremental cost savings of $\$ 8,000$ per QALY gained. Using Fagan's cost saving result at $\$ 600$ per rt-PA treated, it was estimated that in 2005 , for every $2 \%$ increase in the proportion of ischaemic stroke patients receiving rt-PA, the result was $\$ 7$ million dollars of annual healthcare cost savings in the USA [67].

\section{Lowering intraocular pressure to increase optic nerve head perfusion gradient}

\section{Anterior chamber paracentesis}

Complication

This is achieved by inserting a 27 -gauge needle into the anterior chamber via the limbus and withdrawing $0.1 \mathrm{ml}$ to $0.2 \mathrm{ml}$ of aqueous fluid. The potential benefits of paracentesis include a dramatic drop in IOP, and dilatation of the retinal arteries because of the vascular tortuosity resulting from distortion of the globe [49]. However, a maximum increase in retinal arterial volume flow of only $20 \%$ has been estimated from animal studies, and a rise in perfusion pressure of less than $15 \%$ is expected when the IOP falls from $15 \mathrm{mmHg}$ to $5 \mathrm{mmHg}[18,40]$.

Special points The potential risk of paracentesis includes infection.

The procedure is well described and commonly employed [62], but the effectiveness is not proven.

Cost The cost of the procedure is inexpensive. 


\section{Means to lyse and dislodge the embolus}

\section{Nd YAG laser (Neodymium:yttrium-aluminum-garnet laser)}

A single case report has been reported with an improvement of vision following the use of Nd YAG laser in a patient with CRAO. The laser, using 0.8$1.1 \mathrm{~mJ}$ intensity, was focused slightly posterior to the visible arterial wall at the site of the embolus $[68,69]$. The laser tends to dislodge the embolus, which dissolves later. The complication of the laser includes vitreous hemorrhage and false aneurysm. Since these are only case reports and the procedure involves significant complications, YAG laser embolectomy is not accepted as a standard treatment of choice in CRAO.

Pars plana vitrectomy

Complications

Special points

Cost/cost effectiveness

A pars plana vitrectomy followed by removal of the embolus has been shown to be promising in case reports [70,71] and small case series [72]. It has been suggested to perform a fluorescein angiogram to ascertain the site of occlusion, and a longitudinal incision is made over the area of occlusion to remove the embolus. However, the studies are limited and randomised control studies are required to ensure the safety of the treatment of this procedure in CRAO.

Vitreous haemorrhage

Cataract

This procedure is not a standard acute treatment for CRAO.

Expensive when considering the cost of surgery and expertise required. The treatment is not proven, and hence not cost effective.

\section{Neovascularisaion following CRAO}

\section{Treatment of neovascularization}

Neovascularization (Fig. 4) followed by glaucoma occurs in 2.5-31.6\%, and the mean time from CRAO to observed neovascularization was 8.5 weeks (range 216 weeks) [73]. Rudkin et al. reported a definite temporal relationship between the CRAO and neovascularization events in $18.2 \%$ of patients, with no other causes of neovascularization demonstrable in their cohort of patients. In the majority of cases of neovascularisation, there were no clinical features of ocular

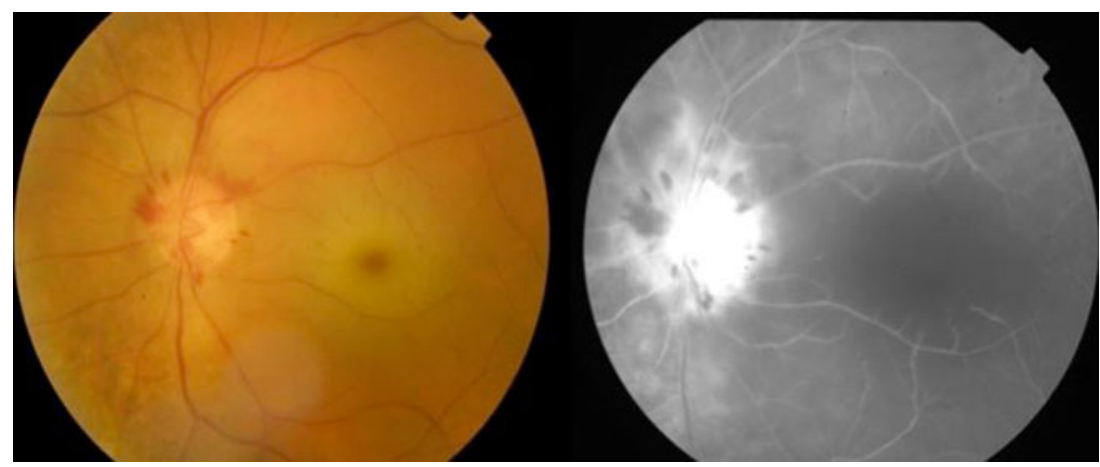

Figure 4. Colour fundus photograph and fundus fluorescein angiogram of the left eye showing neovascularisation of the disc where the new vessel result in leakage and bright hyper-fluorescence at the disc. 


\section{Conclusion}

ischemia, and no association with a hemodynamically significant stenosis of the carotid artery. It is essential to review all patients with acute CRAO at regular intervals as early as 2 weeks, then monthly up to 4 months, post-CRAO. If at any stage, a patient develops neovascularisation, panretinal photocoagulation should be performed promptly. Pan-retinal photocoagulation aims to decrease demand for oxygen in the peripheral retina to reduce the vascular endothelial vascular growth factors that cause abnormal blood vessel. The new vessels, if left untreated, can bleed easily into the eye and result in vitreous hemorrhage or high pressure in the eye (neovascular glaucoma).

CRAO should be considered as an ocular emergency and is the ocular analogue of cerebral stroke. The same atherosclerotic risk factors that predispose to cardio, peripheral and cerebrovascular diseases are present in CRAO, and these must be actively evaluated to prevent further medical comorbidities. Current acute managements in acute CRAO have limited efficacy to improve vision and studies suggest that for treatment to be effective in CRAO, it must be deployed within a short time window, potentially within $6 \mathrm{~h}$ of symptom onset within the retinal tolerance time for any therapy to be effective. Current management follows the same principles of treatment as any other vascular end organ ischaemic disease, and includes vascular review to prevent further end organ ischemia and ocular complications.

\section{Disclosure}

No potential conflicts of interest relevant to this article were reported.

\section{Open Access}

This article is distributed under the terms of the Creative Commons Attribution License which permits any use, distribution, and reproduction in any medium, provided the original author(s) and the source are credited.

\section{References and Recommended Reading}

Papers of particular interest, published recently, have been highlighted as:

- Of importance

- Of major importance

1. Graefes AV. Ueber Embolie der Arteria centralis reti- 3. nae als Ursache plotzlicher Erblindung. Arch Ophthalmol;5:136-157

2. Leavitt JA, Larson TA, Hodge DO, Gullerud RE. The incidence of central retinal artery occlusion in Olmsted County, Minnesota. Am J Ophthalmol. 2011;152:820-3.

This is a large retrospective study which determines the incidence of central retinal artery occlusion.

3. Rumelt S, Brown GC. Update on treatment of retinal arterial occlusions. Curr Opin Ophthalmol. 2003;14:139-41.

4. Hayreh SS, Zimmerman MB. Central retinal artery occlusion: visual outcome. Am J Ophthalmol. 2005;140:376-91.

5. Lorentzen S. Incidence of cilioretinal arteries. Acta Ophthalmol. 1970;48:518-24. 
6.• Hayreh S. Acute retinal arterial occlusive disorders. Progr Retin Eye Res. 2011;30:359-94.

This review article details the anatomy of the blood supply to the eye. The classification of acute retinal arterial ischaemic disorders and their management are discussed.

7. Hayreh S. A Study of the Central Artery of the Retina in Human Beings in its Intra-orbital and Intra-neural Course: Thesis for Master of Surgery, Panjab University, India, 1958. Back to cited text, 1958.

8. Mangat HS. Retinal artery occlusion. Surv Ophthalmol. 1995;40:145-56.

9. Hayreh SS. Prevalent misconceptions about acute retinal vascular occlusive disorders. Progr Retin Eye Res. 2005;24:493-519.

10. Hayreh S, Kolder H, Weingeist T. Central retinal artery occlusion and retinal tolerance time. Ophthalmology. 1980;87:75.

11. Chen CS, Lee AW. Management of acute central retinal artery occlusion. Nat Clin Pract Neurol. 2008;4:376-83.

12. Rudkin A, Lee A, Chen C. Vascular risk factors for central retinal artery occlusion. Eye. 2009;24:678-81.

13. Schmidt DP, Schulte-Mönting J, Schumacher M. Prognosis of central retinal artery occlusion: local intraarterial fibrinolysis versus conservative treatment. Am J Neuroradiol. 2002;23:1301-7.

14. Kline L. The natural history of patients with amaurosis fugax. Ophthalmol Clinics of $\mathrm{N} \mathrm{Am}$. 1996;9:351-8.

15. Savino PJ, Glaser JS, Cassady J. Retinal stroke: is the patient at risk? Arch Ophthalmol. 1977;95:1185.

16. Augsburger J, Magargal L. Visual prognosis following treatment of acute central retinal artery obstruction. Br J Ophthalmol. 1980;64:913-7.

17. Pfaffenbach D, Hollenhorst R. Morbidity and survivorship of patients with embolic cholesterol crystals in the ocular fundus. Trans Am Ophthalmol Soc. 1972;70:337.

18. Ffytche T. A rationalization of treatment of central retinal artery occlusion. Trans Ophthalmol Soc U K. 1974;94:468-79.

19. Wang FM, Henkind P. Visual system involvement in giant cell (temporal) arteritis. Surv Ophthalmol. 1979;23:264-71.

20. McBrien D, Bradley R, Ashton N. The nature of retinal emboli in stenosis of the internal carotid artery. Lancet. 1963;281:697-9.

21. Cogan DG, Kuwabara T, Moser H. Fat emboli in the retina following angiography. Arch Ophthalmol. 1964;71:308-13.

22. David NJ, Lintworth GK, Friedberg SJ, Dillon M. Fetal atheromatous cerebral embolism associated with bright plaques in the retinal arterioles. Report of a case. Neurology. 1963;13:708-13.
23. Greven CM, Slusher MM, Weaver RG. Retinal arterial occlusions in young adults. Am J Ophthalmol. 1995; 120:776-83.

24. Schmidt D, Hetzel A, Geibel-Zehender A, SchulteMonting J. Systemic diseases in non-inflammatory branch and central retinal artery occlusion-an overview of 416 patients. Eur J Med Res. 2007;12:595603.

25. Hayreh SS, Piegors DJ, Heistad DD. Serotonin-induced constriction of ocular arteries in atherosclerotic monkeys: implications for ischemic disorders of the retina and optic nerve head. Arch Ophthalmol. 1997;115:220-8.

26. Hjermann I, Velve BK, Holme I, Leren P. Effect of diet and smoking intervention on the incidence of coronary heart disease. Report from the Oslo Study Group of a randomised trial in healthy men. Lancet. 1981;2:1303-10.

27. Pearson TA, Blair SN, Daniels SR, et al. AHA guidelines for primary prevention of cardiovascular disease and stroke: 2002 update consensus panel guide to comprehensive risk reduction for adult patients without coronary or other atherosclerotic vascular diseases. Circulation.

2002;106:388-91.

28. Sottilotta G, Oriana V, Latella C, et al. Role of hyperhomocystinemia in retinal vascular occlusive disease. Clin Appl Thromb Hemost. 2007;13:104-7.

29. Hayreh SS, Zimmerman MB, Kimura A, Sanon A. Central retinal artery occlusion: retinal survival time. Exp Eye Res. 2004;78:723-36.

30. Chen CS, Lee AW, Campbell B, et al. Efficacy of intravenous tissue-type plasminogen activator in central retinal artery occlusion. Stroke. 2011;42:2229-34.

This study highlights that intravenous tPA has minimal effect in patients diagnosed to have CRAO, especially if administered after the window period of 6 hours. The study also highlights the increased risk of reocclusion in these patients.

31. Hattenbach LO, Kuhli-Hattenbach C, Scharrer I, Baatz $\mathrm{H}$. Intravenous thrombolysis with low-dose recombinant tissue plasminogen activator in central retinal artery occlusion. Am J Ophthalmol. 2008;146:700-6. e701.

32. Beatty S, Eong KGA. Local intra-arterial fibrinolysis for acute occlusion of the central retinal artery: a meta-analysis of the published data. Br J Ophthalmol. 2000;84:914-6.

33. Landa E, Rehany U, Rumelt S. Visual functions following recovery from non-arteritic central retinal artery occlusion. Ophthalmic Surg Laser Imag. 2004;35:103.

34. Mueller AJ, Neubauer AS, Schaller U, Kampik A. Evaluation of minimally invasive therapies and rationale for a prospective randomized trial to evaluate selective intra-arterial lysis for clinically complete 
central retinal artery occlusion. Arch Ophthalmol. 2003;121:1377-81.

35. Incandela L, Cesarone M, Belcaro G, et al. Treatment of vascular retinal disease with pentoxifylline: a controlled, randomized trial. Angiology. 2002;53:31-4.

36. Fraser SG, Adams W. Interventions for acute nonarteritic central retinal artery occlusion. Cochrane Database Syst Rev 2009;1.

37. Arend O, Harris A, Martin BJ, Holin M, Wolf S. Retinal blood velocities during carbogen breathing using scanning laser ophthalmoscopy. Acta Ophthalmol. 1994;72:332-6.

38. Harino S, Grunwald JE, Petrig BJ, Riva CE. Rebreathing into a bag increases human retinal macular blood velocity. Br J Ophthalmol. 1995;79:380-3.

39. Deutsch TA, Read JS, Ernest JT, Goldstick TK. Effects of oxygen and carbon dioxide on the retinal vasculature in humans. Arch Ophthalmol.

1983;101:1278-80.

40. Atebara NH, Brown GC, Cater J. Efficacy of anterior chamber paracentesis and Carbogen in treating acute nonarteritic central retinal artery occlusion. Ophthalmology. 1995;102:2029-34. discussion 2034-2025.

41. Aisenbrey S, Krott R, Heller R, Krauss D, Rössler G, Heimann K. Hyperbaric oxygen therapy in retinal artery occlusion. Ophthalmologe: Zeitschrift der Deutschen Ophthalmologischen Gesellschaft. 2000;97:461-7.

42. Menzel-Severing J, Siekmann U, Weinberger A, Roessler G, Walter P, Mazinani B. Early hyperbaric oxygen treatment for nonarteritic central retinal artery obstruction. Am J Ophthalmol. 2012;153:454-9.

43. Cope A, Eggert J, O'Brien E. Retinal artery occlusion: visual outcome after treatment with hyperbaric oxygen. Diving Hyperb Med. 2011;41:135-8. This case series discusses the visual outcome of patients treated with hyperbaric oxygen therapy in CRAO.

44. Hertzog L, Meyer G, Carson S, Strauss M, Hart G. C treated with hyperbaric oxygen. Undersea Hyperb Med. 1992.

45. Weiss JN. Hyperbaric oxygen treatment of nonacute central retinal artery occlusion. Undersea Hyperb Med. 2010;37:167-72.

46. Anderson Jr B, Saltzman HA, Heyman A. The effects of hyperbaric oxygenation on retinal arterial occlusion. Arch Ophthalmol. 1965;73:315-9.

47. Butler Jr F, Hagan C, Murphy-Lavoie H. Hyperbaric oxygen therapy and the eye. Undersea Hyperb Med. 2008:333-387

48. Rumelt S, Dorenboim Y, Rehany U. Aggressive systematic treatment for central retinal artery occlusion. Am J Ophthalmol. 1999;128:733-8.

49. Beatty S, Eong KGA. Acute occlusion of the retinal arteries: current concepts and recent advances in di- agnosis and management. Emerg Med J. 2000;17:324-9.

50. Hausmann N. High dose steroid bolus given for occlusion of central retinal artery: author's reply. $\mathrm{Br}$ Med J. 1992;304:506-7.

51. Pandit JC, Tiamiyu F. High dose steroid bolus given for occlusion of central retinal artery. BMJ: Br Med J. 1992;304:506-7.

52. Biousse V, Calvetti O, Bruce BB, Newman NJ. Thrombolysis for central retinal artery occlusion. J Neuro Ophthalmol. 2007;27:215-30.

53. Noble J, Weizblit N, Baerlocher M, Eng K. Intra-arterial thrombolysis for central retinal artery occlusion: a systematic review. Br J Ophthalmol. 2008;92:588-93.

54. Aldrich EM, Lee AW, Chen CS, et al. Local Intraarterial fibrinolysis administered in aliquots for the treatment of central retinal artery occlusion. Stroke. 2008;39:1746-50.

55. Zhang X, Ji X, Luo $Y$, et al. Intra-arterial thrombolysis for acute central retinal artery occlusion. Neurol Res. 2009;31:385-9.

56.• Schumacher M, Schmidt D, Jurklies B, et al. Central retinal artery occlusion: local intra-arterial fibrinolysis versus conservative treatment, a multicenter randomized trial. Ophthalmology 2010;117:13671375. e1361.

This randomised multicentre study compares the treatment outcomes of conventional treatment versus intra-arterial tPA in patients with CRAO.

57. Lindsberg PJ, Mattle HP. Therapy of basilar artery occlusion a systematic analysis comparing intra-arterial and intravenous thrombolysis. Stroke. 2006;37:922-8.

58. Arnold M, Koerner U, Remonda L, et al. Comparison of intra-arterial thrombolysis with conventional treatment in patients with acute central retinal artery occlusion. J Neurol Neurosurg Psychiatr. 2005;76:196-9.

59. Biousse V. Thrombolysis for acute central retinal artery occlusion: is it time? Am J Ophthalmol. 2008; 146:631.

60. Feltgen N, Neubauer A, Jurklies B, et al. Multicenter study of the European Assessment Group for Lysis in the Eye (EAGLE) for the treatment of central retinal artery occlusion: design issues and implications. EAGLE Study report no. 1. Graefes Arch Clin Exp Ophthalmol. 2006;244:950-6.

61. Genentech. (2011). Activase, Alteplase, a recombinant tissue plasminogen activator. Available: http:// www.gene.com/gene/products/information/ cardiovascular/activase/. Last accessed 16/8/12.

62. Derex L, Nighoghossian N. Intracerebral haemorrhage after thrombolysis for acute ischaemic stroke: an update. J Neurol Neurosurg Psychiatr. 2008;79:1093. 
63. PDR Staff. 2004 Red Book Pharmacy's Fundamental Reference. 2004th ed. Montvale: Thomson Healthcare; 2004. p183.

64. Fagan S, Morgenstern L, Petitta A, et al. Cost-effectiveness of tissue plasminogen activator for acute ischemic stroke. Neurology. 1998;50:883-90.

65. Demaerschalk BM, Hwang HM, Leung G. Cost analysis review of stroke centers, telestroke, and rt-PA.

Am J Manag Care. 2010;16:537.

66. Tissue plasminogen activator for acute ischemic stroke. The National Institute of Neurological Disorders and Stroke rt-PA Stroke Study Group. N Engl J Med 1995;333:1581-1587.

67. Demaerschalk BM, Yip TR. Economic benefit of increasing utilization of intravenous tissue plasminogen activator for acute ischemic stroke in the United States. Stroke. 2005;36:2500-3.

68. Reynard M, Hanscom TA. Neodymium: yttrium-aluminum-garnet laser arteriotomy with embolectomy for central retinal artery occlusion. Am J Ophthalmol. 2004;137:196-8.

69. Haritoglou C, Wolf A, Ulbig MW. Laser embolectomy for central retinal artery occlusion. Ophthalmologe. 2010;107:465-7.

70. Tang WM, Topping TM. Vitreous surgery for central retinal artery occlusion. Arch Ophthalmol. 2000;118: 1586-7.

71. Peyman GA, Gremillion CM. Surgical removal of a branch retinal artery embolus: a case report. Int Ophthalmol. 1990;14:295-8.

72. Garcia-Arumi J, Martinez-Castillo V, Boixadera A, Fonollosa A, Corcostegui B. Surgical embolus removal in retinal artery occlusion. Br J Ophthalmol. 2006;90:1252-5.

73. Rudkin AK, Lee AW, Chen CS. Ocular neovascularization following central retinal artery occlusion: prevalence and timing of onset. Eur J Ophthalmol. 2010;20:1042-6. 